\title{
THE ROLE OF INFORMATION INSTRUMENTS IN ENSURING TRANSPARENCY IN SCIENTIFIC RESEARCH (THE CASE OF THE REP. OF MOLDOVA)*
}

\author{
Holban Ion ${ }^{1}$, Cuciureanu Gheorghe ${ }^{2}$ and Minciuna Vitalie ${ }^{3}$
}

\begin{abstract}
The present paper shows several information instruments created in the Republic of Moldova for the purpose of ensuring transparency in science, innovation and scientific staff training. Their evolution, the way in which they could help enhancing transparency in Moldovan researchdevelopment-innovation system, shortcomings in ensuring greater visibility, have been investigated. Three information instruments developed (or under preparation) by the Information Society Development Institute have been examined in detail: i) National Bibliometric Instrument - a digital information system which is a database for collecting, classifying and processing public data on scientific publications in Moldovan scientific journals; ii) EXPERT online - an online information system for applying and assessment of draft programs and projects in the area of science and innovation; iii) Digital Map of Science - a platform in which the functions of existing systems are to be integrated under a common roof in order to provide one-stop-shop services.
\end{abstract}

Key words: transparency, information systems, open science, digital content, research management

\section{Introduction}

The information instruments give more opportunities for enhancing the transparency of scientific activities and contribute to the quality growth of the obtained results. This is provided by both the increased access to scientific information and the decision making process (selection of the projects, financial distribution, granting awards, scientific degrees etc.). In this way, transparency may contribute to a higher efficiency in using the resources.

The transparency of scientific content fits on the concept of Open science, the importance of which is widely acknowledged in the world. Thus, transparency is a basic element of the Horizon-2020 framework program of the European Commission and 4 global scientific organisations (the International Council for Science, the InterAcademy Partnership, the World Academy of Sciences and the International Social Science Council) stipulate in an international agreement, that opening and transparency are the main elements for the progress of science nowadays [6].

However, the information instruments can't assure transparency by their own and their impact may differ in different contexts. Their success in the ensuring of transparency and the prevention of

\footnotetext{
*This research was made within the project 15.817.06.13.A "Pilot platform for ensuring the evaluation of quality and the visualisation of scientific digital content in the Republic of Moldova" (2015-2018), http://idsi.md/sciform ${ }^{1}$ National Council For Accreditation and Attestation, Stefan cel Mare bd., 180, Chisinau, MD-2004; Information Society Development Institute, 5A Academiei, Chisinau, MD-2028, Republic of Moldova, ion.holban@yahoo.com ${ }^{2}$ Information Society Development Institute, 5A Academiei, Chisinau, MD-2028, Republic of Moldova, gheorghe.cuciureanu@idsi.md

${ }^{3}$ Information Society Development Institute, 5A Academiei, Chisinau, MD-2028, Republic of Moldova, vitalie.minciuna@idsi.md
} 
deviations from the norms of right conduct depends on the way of implementation, education and acceptance by the citizens etc. [1].

Transparency in research-development is closely linked to the concepts of digital science and Science 2.0, which presuppose new ways of research (use of web technologies, computer resources, research and analytical instruments etc.) and of evaluation of the research results (webometrics, altmetrics). In the European Union, the goal is to support as much as possible digital science in order to assure more opportunities for science and society. As a whole, the opening up access to the results and processes of research by digital means and the involvement of people as active participants in the processes of research, stimulate the development of ethical and responsible research models if the citizens and society contribute to the results and shape the process of research [5].

\section{Issues of ensuring transparency in scientific research in the Republic of Moldova}

Until 1991, the Republic of Moldova was part of the Soviet Union. Many key functions, such as the development of science and the training of scientific staff, were directed by Moscow. After the declaration of independence, the Republic of Moldova was trapped into a long transitional period from a planned economy to free market, which had an impact on all fields of activity, including the research-development. Currently, one of the biggest problems is the lack of transparency and the corruption. According to Transparency International, in 2015 the Republic of Moldova was the $103 \mathrm{rd}$ in this matter [7].

The current situation in the field of research-development is a consequence of several factors, both objective (economic collapse, scarce funding of research, exodus of experts) and subjective ones (incapacity or unwillingness to learn about new organisational forms of the research process, based on principles of competitiveness; lack of qualified experts or random selection of experts; no incentives for the experts etc.). Although after the year 2000, the competition became a normal element in the field of research-development, the objectivity of the evaluation, of the selection of the most performant projects, of the distribution of financial resources still remains a problem. This happens because of the small size of the scientific community and the lack of mechanisms to promote meritocracy.

In order to make the evaluation objective and to assure the transparency of the process, it was imposed to create databases regarding the existent scientific potential and transparency-improving instruments for the research institutions. Several attempts of elaborating some functional platforms regarding this cause were made, but the structural reorganisations of the institutions responsible for these activities and the lack of a normative, coherent framework regarding the maintenance, the teaching-reception of the databases by activity fields, made these attempts unsuccessful.

Simultaneously, the intensification of the use of information technologies into the solving process of diverse problems, including the assurance of transparency of scientific activities, is assumed at national level by:

- $\quad$ Moldova-European Union Association Agreement (chapter 18 and 24), and by relevant national strategies:

- “Digital Moldova 2020” Strategy (pylon II - Digital content and electronic services), 
- "Strategy of research-development until 2020" (section 5: Ensuring of information support of the project evaluation; building of digital content based on the results of scientific research and its dissemination through the use of the TIC solutions),

- "National Program of computerisation of the cultural sphere 2012-2020" (section 2:

Digitisation of the material and immaterial cultural patrimony).

Taking into account the state of affairs, but also considering the role of transparency for the improvement of the situation, we want to see how the most important information instruments contribute to the transparency ensuring of scientific activities in the Republic of Moldova. We analysed the websites of the most important actors and the specialised information instruments with the highest impact. The analysis of the instruments has been conducted according to the following methodological scheme: 1) the instrument's key features - 2) its role within the RDI system and in ensuring transparency - 3) the instrument's strong and weak points in ensuring transparency - 4) its impact on ensuring transparency and on well-functioning of the national RDI system. Even though it was not always possible to fully elucidate all these aspects, we think that the key elements which were highlighted, allow us to observe their effect on the system's transparency.

\section{The main information instruments that contribute to transparency ensuring in scientific research in the Republic of Moldova}

\subsection{The websites of the institutions that administrate/coordinate scientific activities}

\subsubsection{The website of the Academy of Science of Moldova - http://asm.md/}

The Academy of Science of Moldova (ASM) has the main organisational role in the field of science in the Republic of Moldova. After concluding of a partnership agreement with the government, the functions of elaboration of policy in this field are delegated to the Academy, which therefore has the role of a science ministry. ASM is also the most important body for politics implementation: it is a finance agency of research and innovation (administrating almost all public resources in the field of science through its agencies), it is the main organisation doing research at national level and it has its own university. From here arises the outstanding importance for transparency ensuring of research-development activity at national level.

The ASM website is a traditional one, with rubrics characteristic for both a public authority and a scientific structure. Although it is the only site which could have synthesised and presented relevant research information, it is a static one, without any information instruments supplying automatically synthesised data. For example, there is a "Scientific researchers" rubric, but when one tries to click on it, it turns out to be a redirection to the respective rubric on Expert-online.

An important rubric of an authority in this field is "Regulatory framework". Unfortunately, the decisions of the Supreme Council for Science and Technological Development (SCSTD) (executive body of ASM) are placed selectively. Moreover, elaborated normative acts and acts waiting for approval are not placed on the website for discussions.

There also exist "satellite" sites of the subordinate administration agencies/structures (e.g.: http://www.acd.asm.md/, http://cpi.asm.md/, http://aitt.md/). Besides the fact that they take some information from the main ASM website, they also publish original material. They neither do not have incorporated specific information instruments for calls and online sending of project 
proposals, evaluation, project management or result reporting. In fact, they do not contain databases. Overall, these websites give a general image of the national system of researchdevelopment, but there aren't interactive possibilities or instruments for generating information requested by the users. However its impact is a major one. In the last (January 2017) edition of Webometrics - Ranking Web of Research Centres, the Academy of Sciences of the Republic of Moldova ranks $25^{\text {th }}$ in Central and Eastern Europe ( $659^{\text {th }}$ worldwide).

\subsubsection{The website of the National Council for Accreditation and Attestation - http://www.cnaa.md/}

The National Council for Accreditation and Attestation (NCAA) has an important role in the national research-development system design because, on one hand, it confers scientific and scientific-didactic titles and degrees and on the other hand, it accredits and evaluates scientific organisations. In the Republic of Moldova, only organisations accredited by the NCAA may benefit of funding from the state scientific research budget.

The website of the NCAA includes all decisions of the institution in the field of attestation and accreditation, assuring the access for the users to the data regarding the evaluation of staff and organisations. Whereas at the beginning of the website development the institution had no responsibilities in the field of accreditation (which have been attributed in 2005), the site assures a high degree of transparency and interconnection of data regarding the attestation of scientific staff. There is information regarding the preparation of scientific frameworks (nomenclature of scientific specialities, institutions organising the framework preparation, doctorate commissions - specialised scientific councils, profile scientific seminars and $\mathrm{PhD}$ theses) and the existent scientific potential ( $\mathrm{PhD}$ supervisors, holders of scientific degrees, $\mathrm{PhDs}$ and post-PhDs). The website is based on relations between content knots. One of the used mechanisms is the contextual filtration method, used for the implementation of alternative means of site navigation. The application of this method accentuates the link between the site and other parts of the site and contributes to the awareness of the site content position in the information space of the site. [2].

An important element is the embedding of different elements of the thesis defending, the scientific degree granting and the interconnection between these two processes. In fact, the rubric dedicated to the theses is the most dynamic one on the website, because in the Republic of Moldova, 200 theses are defended every year. The elements of the thesis (title, author, degree, scientific field, speciality, date, scientific supervisor, official referents, the institution where the thesis was elaborated, the doctorate commission, key words, annotations, summary in pdf-form, the thesis in pdf-form) are kept separately and are interconnected. Thanks to this, the thesis is represented by relations with other knots, giving the possibility to search/filter according to several parameters. Thus, apart from the thesis in pdf-form (which is very important for transparency ensuring in the scientific degree granting process as it can be evaluated by everyone), we can see the repartition of the theses by institutions, specialities, scientific supervisors etc. A particular importance for ensuring transparency and preventing cheating comes to a statistic regarding the links between different persons participating in the process of thesis taking (between the $\mathrm{PhD}$ supervisor and the members of the doctorate commission, between different members etc.), thus finding the existent conflict of interests.

The introduction of the instrument, by placing doctoral theses in Open access (since 2005), as well as the online broadcasting of doctoral theses defenses (since 2013), has contributed to a quality improvement of the theses and to a reduction of scientific frauds, but also helped involving some foreign experts in evaluation of theses. Thus, while an average of 108 foreign experts participated in 
doctoral committees in 2007-2010, the figure has grown up to 144 in the years 2013-2016, which is $17.5 \%$ of the total number of doctoral committees' members.

It is noteworthy that NCAA, by its theses database, is the only national repository of the Republic of Moldova in the Register of Open Access Repositories (ROAR), being included in 2010, therefore enhancing the transparency and allowing the execution of bibliometric studies. Simultaneously, there are some shortcomings of the NCAA website which decrease the impact of transparency: the annual rapports of NCAA are not posted, the $\mathrm{PhD}$ theses are posted only for a limited period of time (only the summaries are permanent), with the change of the nomenclature of scientific specialities in the Republic of Moldova, the website hasn't yet the capacities of presenting the information according to the new standards, there is little information regarding the decline of scientific degree requests, what doesn't discourage people from making deviations from the rules of good conduct in research, there isn't a theses discussion forum, that could be interesting and enhance the transparency in the process of $\mathrm{PhD}$ defending.

\subsubsection{The website of the State Agency for Intellectual Property - http://agepi.md/}

The State Agency for Intellectual Property (AGEPI) is the main public authority in the Republic of Moldova in the field of intellectual property protection (intellectual property objects (IPO), author rights). Besides the protection ensuring and the legal interests of the authors, AGEPI has the attributions to develop and enforce the national system of intellectual property, to fight and prevent the violation of rights of intellectual property and to fight counterfeit and piracy.

The AGEPI website is a complex one, with good interaction between different components. This allows the presentation of diverse information to a diverse audience. All aspects regarding intellectual property that could be of interest for the public are covered, for professional purposes or just for curious users. The users are faced to very detailed information, accompanied by online services and application mechanisms (using digital signature), for brand and industrial model protection, the protection of inventions, plants, literary, scientific, musical or art work, but also for making documented researches.

The transparency regarding the activity of intellectual property protection is very good. The website incorporates a lot of well structured databases (fig.1). All of the 9 existent databases allow the search and display of information regarding intellectual property objects (IPO) by several parameters/criteria. Moreover, there are instruments that generate certain rapports. Thus, for inventions, the National patent landscape is generated, which presents generalised information regarding the patent activity at national level, and the Patent Profile of the Person (inventor/applicant/patent owner), which allows for performing a multilateral analysis of every person's patent activity. The information generated by these instruments is very interesting, both for a large public and for decision-makers.

The information solutions incorporated on the AGEPI website allow a detailed monitoring of the process of intellectual property ensuring and a real opening at a large spectrum of users. In 2016 the number of online applications for registration or renewal of IPO doubled comparing to last year, thus reaching $42 \%$ of the total. Transparency of the patenting procedure provided for this indicator's large scale utilization in the procedure of scientific accreditation of institutions, especially that the website automatically generates different ratings for individuals and legal entities. The stimulation of the creative activity through the AGEPI website could be regarded as a 
contribution to Moldova ranking the $46^{\text {th }}$ worldwide, before some countries in the region, like Romania, Ukraine and Croatia, according to The Global Innovation Index 2016.

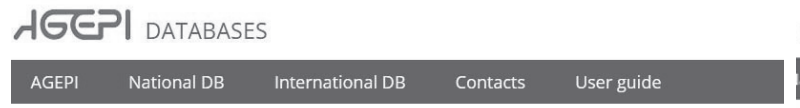

\section{DATABASES}
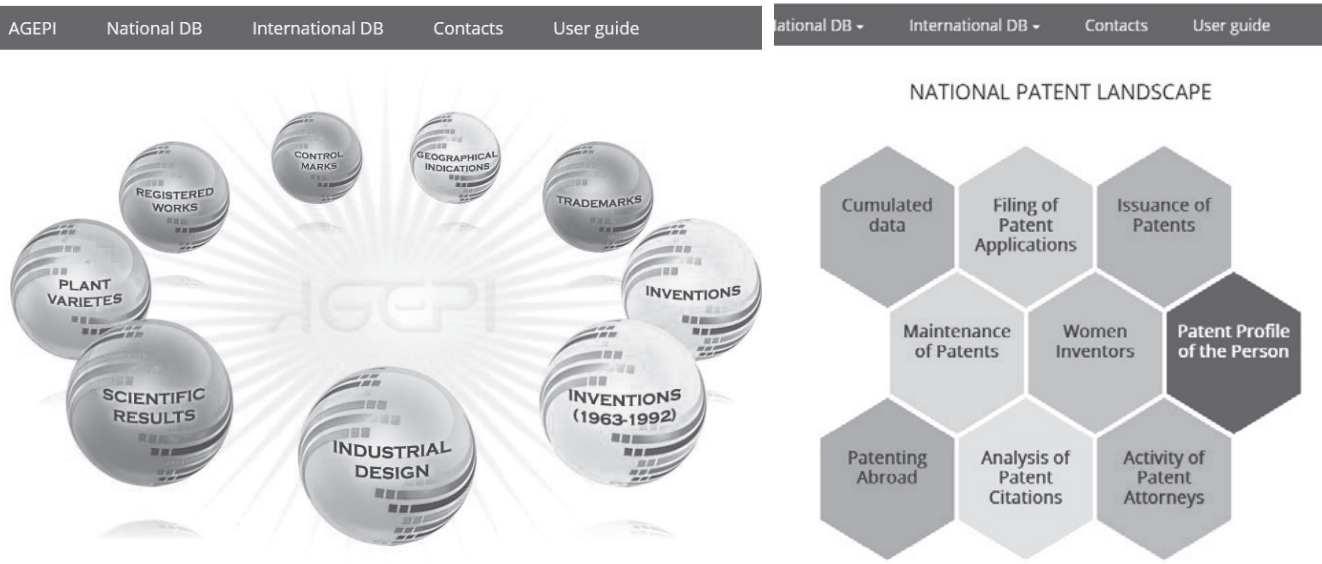

Figure 1: Examples of sites/interfaces of databases on the AGEPI website

Source: http://www.db.agepi.md/

\subsection{Specialised informatics instruments}

\subsubsection{National Bibliometric Instrument - http://ibn.idsi.md/}

The National Bibliometric Instrument (IBN), elaborated by the Information Society Development Institute (ISDI), represents an electronic scientific library regarding the scientific contributions of researchers in the Republic of Moldova. That contributes to the transparency ensuring of research results [3]. The project started in 2010 and today it includes articles published in scientific journals in the Republic of Moldova. The instrument offers the possibility to visualise and download the content of all scientific articles from the system, moreover, it provides information regarding the journals where the articles have been published, author names, the projects, the institutions, the countries of the authors and the scientific fields to which they belong. This information, taken from over 100 journals, is given dynamically (tab.1).

IBN also assures transparency in the process of making the decision if a journal should be accredited in the Republic of Moldova (in order to be considered as scientific, the journals have to satisfy a lot of criteria, the fulfillment of the criteria is verified by an evaluation commission, the SCSTD and NCAA making the decision of accreditation (with the attribution of the category A, B and $\mathrm{C}$ or the decision to not accredit). On one hand, the objectivity of the commission can be verified, on the other hand, because of the keeping of all evaluation and accreditation decisions, the evolution of every journal can be followed.

The transparency of the articles in the national journals has both a utilitarian role, as it offers possibilities to the researchers to find the needed information in one place, and a role of support for the evaluation and the decision making process in the scientific field. Simultaneously, the quality of the publications increases, as both the authors and the editorial boards of the scientific journals are 
more careful about the publications that become a public good for all users. Thanks to the available application, the transparency for the collaboration with other authors is also provided (fig.2).

\begin{tabular}{|l|l|l|l|}
\hline \multicolumn{1}{|c|}{ Content } & Number & \multicolumn{1}{c|}{ Content } & Number \\
\hline Included accredited journals & 64 & Number of journals & 2212 \\
\hline Category A journals & 5 & Articles & 49152 \\
\hline Category B+ journals & 1 & Unique authors & 24500 \\
\hline Category B journals & 28 & Institutions & 2328 \\
\hline Category C journals & 30 & Downloaded articles & 39370 \\
\hline Unacknowledged journals & 38 & Number of downloaded journals & 1602 \\
\hline
\end{tabular}

Table 1: The content of the National Bibliometric Instrument

Source: IBN, on the 23th of February 2017

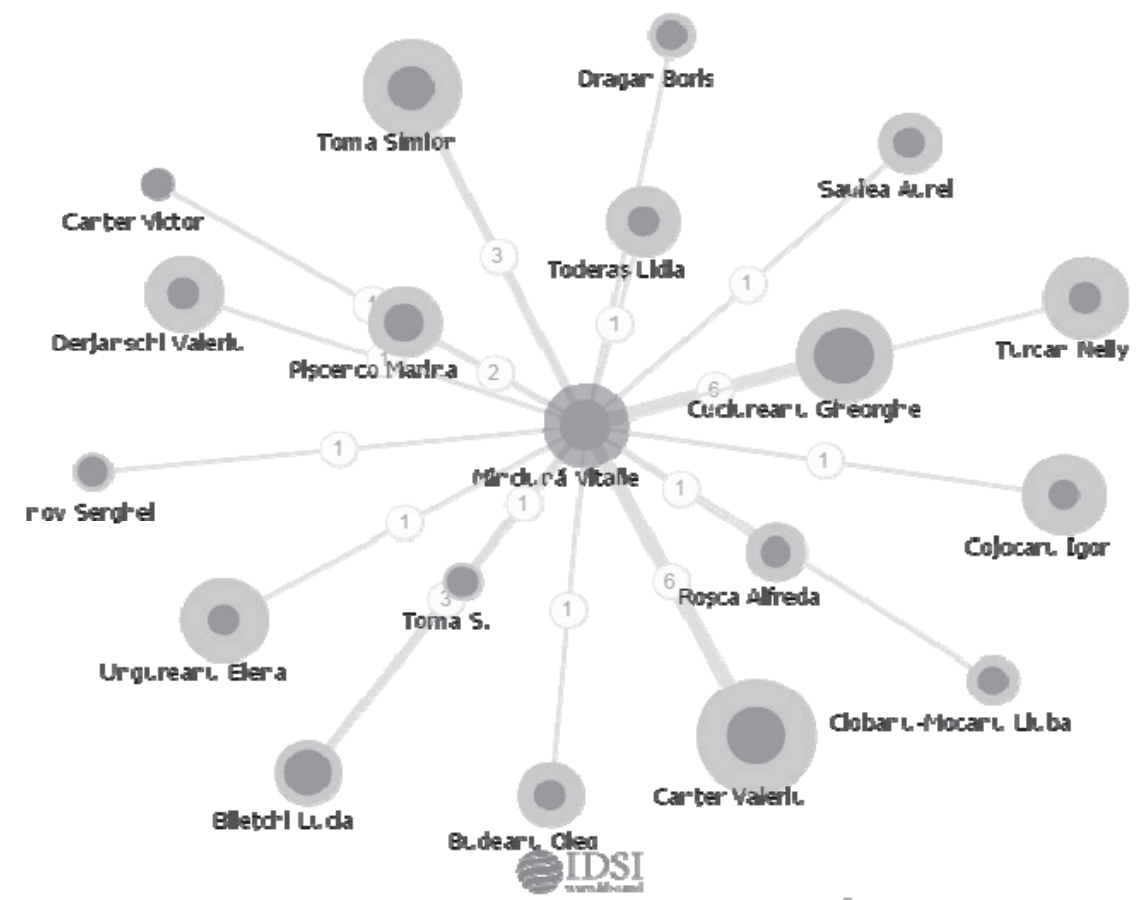

Figure 2: Example of transparency assurance of collaborations in the IBN

Source: http://ibn.idsi.md/ro/displayGraph_page?id=25636

The IBN impact is reflected in the dynamics of the number and quality of scientific journals in the country. Thus, the number of accredited journals droped from 77 in 2013, to 64 in 2016, including due to monitoring through the IBN of the publication frequency, of the compliance with technical requirements and rules of good conduct in publishing (some journals which allowed plagiarism were excluded). At the same time, the number of higher quality journals (A class) increased from 1 to 5 due to their access to the most important international databases, like Thomas Reuters Web Of 
Science and Elsevier Scopus. The number of journals from the Republic of Moldova in the D.O.A.J. increased up to 17, of which 11 being registered during last two years.

The impact is limited because not all publications of Moldovan researchers are included in this database. The pieces of work from abroad and some types of work published in the Republic of Moldova are missing (conference proceedings, monographs etc.). Simultaneously, the introduced metadata do not always permit the finding of the searched information. Thus, the bibliographic references are not included separately into the database and at the moment, citations cannot be followed. Currently, ISDI works both on the bringing in the system of missing publications and on the increasing of the number of publications. Other causes that decrease the effect of IBN as a transparency instrument of scientific activity are: lack of compulsoriness for including the publication into a database in order to be taken into consideration at the reporting, delay of the editing of publications by some journals, delayed transmission of a journal publication to IBN, nonconformation to the demands of the Regulation of evaluation and accreditation of journals. We shouldn't forget the general level of transparency culture in the Republic of Moldova either.

\subsubsection{Expert-online - https://expert.idsi.md/}

The platform Expert-online was designed by the ISDI (2009-2010) initially as an information system for reception and evaluation of projects proposals in the field of innovation and science. At the debut, it represented a source of independent data, regarding the existing human resources, then it was extended by the taking of the registers that reference to the training and attestation process of scientific frameworks and of research projects in the field of science, then it was interconnected with IBN and the AGEPI website. This allowed the import of IPO from AGEPI. The registers of human potential with scientific degrees and scientific-didactic degrees and titles, the registers of journals, projects, organisations allowed the search and displaying of information by diverse criteria. Thus, the register of persons with scientific degrees and scientific and scientific-didactic degrees and titles allows the generation of data regarding the repartition of persons according to the held title or degree, by organisations, fields of research, specialities, a statistic that is unique in the Republic of Moldova and is necessary for scientific research.

Having as an initial goal to make the expert selection process more objective, the mentioned instruments have significantly contributed to the rise of transparency of the decision making process, showing the competency level of the experts, at the level of argumentation for the distributed financial means volume, cost-efficiency relations etc. ISDI has taken into its responsibility to maintain and develop these platforms of documentation in the field of science and innovation, but this task makes it necessary to involve other persons, holders of information or persons responsible for the activity management in the field of science.

An example for the impact of information instruments is the relation of the informational instruments with the process of scientific accreditation of organisations. In the Republic of Moldova, at least at the early stage, the adoption of activity standards and extern evaluation standards has imposed a higher transparency level on the real situation in the institutes.

The evaluation of the institutes has allowed to intervene with corrections and to develop the database platforms in the field of science and innovation. Among the scientific accreditation standards stipulated by the regulatory framework there also is the requirement of the involvement of the organisation into the editing process of the scientific journals. This element provided the possibility of an approach of the problems regarding the editing of the scientific journals at another 
level, of promotion of Open access policies to the scientific results achieved by public financing. The requirement to obligatorily adopt scientific journals by the IBN, laid down by additional documents, has contributed to the development of the IBN and to the expansion of scientific and information possibilities of this instrument.

IBN has assured a certain level of transparency on the scientific results, but the situation regarding resources in the field of science and innovation is elucidated by Expert-online. The procedure of scientific accreditation has allowed the evaluation of human potential entrained into the RDI activities, the level of qualification, the involved organisations, the applied logistics, the national and international projects. The undertaken evaluation activities have made it possible to intervene with corrections and supplements for the platform Expert-online, specifying the held resources in the public sector, and to make proposals for the change of the organisation financing principles, in conformity with the demonstrated performance. We can assess, that Expert-online made the selection procedure of the NCAA and SCSTD experts more objective, being now applied also in drafting efficiency ratings of the scientific personnel.

\subsubsection{The digital map of science}

In order to enhance the quality of the available digital services, the ISDI has started a project for integration at data level in 2015, based on the scientific-information network, on the existent information systems that function separately (IBN, Expert-online, Indicatori-2020, E-Rating etc.), on a new platform. The new platform is based on the CERIF standards [4], which will have the digital map of science as an interface. The platform tends to complete the information articulation between the stage of launch of creative activities and the stage of publication of the digital content and the offering of information support for the application of the criteria of scientific quality into the financing decisions and into the important evaluations of scientific literature. The realisation of this instrument is based on the following hypotheses:

(1) the production of digital scientific content is a framework for the development of the information society in the Republic of Moldova;

(2) the Open access ensures the transfer of new scientific knowledge to other scientific branches, society and application of these in economics, education, culture and public administration;

(3) quality and intern and international visibility of digital scientific content ensuring favours the development of the information society in the Republic of Moldova on the routes that are in concordance with the Association Accord of the Republic of Moldova to the European Union.

The platform should be an information support for the creators and beneficiaries of digital scientific content in the Republic of Moldova regarding: a) the assurance of quality into the creation of digital scientific content and b) the enhancement of content visibility by Open access.

The innovations of the platform are: supply of a variety of information, visible at national and international level regarding the RDI, that will lead to a higher transparency of the activities in the field of science and innovation, with the following consequences: 
- $\quad$ the area of dissemination of new knowledge will increase;

- technical facilities and the fact that the access to digital content is free favour the appliance of research results in industry, education, culture and other activity fields;

- $\quad$ the implementation of other national registers in the RDI field will be ensured, including the reutilisation of information based on the interoperability framework in the RDI field;

- collaboration and efficient communication between institutional actors and professional communities from the country will be facilitated in order to design and apply public policy regarding the development of the information society in the context of European integration.

\section{Conclusions}

The study showed that the information instruments are important and necessary in ensuring transparency in RDI. In the conditions of the Republic of Moldova, the instruments can help overcoming specific problems, issued from the fact that the Republic of Moldova is a post-soviet country (with a mentality and organisation that leads to a high degree of corruption), with a small scientific community, where there are strong relationships between its members, also of relatedness, and a small number of honest and competent experts.

It is hard to quantitatively evaluate the utility of the described instruments, but the general perception and some results/changes observed upon their introduction, indicate that they play a positive role in respecting rules of good conduct in research and professional training of scientific staff.

The impact of the instruments is limited because of the low level of transparency culture in the local scientific community and because of the lack of functioning of some mechanisms. These lead to the fact that some scientific frauds or arbitrary administrative decisions, that can be established by information instruments, are ignored. This demotivates honest behaviour. In this context, it is highly necessary to develop/enhance transparency culture in RDI in the Republic of Moldova.

The increase of the degree of transparency in national science is necessary, both for enhancing the quality of scientific production and for national goals, regarding the integration of the Republic of Moldova into the European Research Area and the European Area of Higher Education, as elements of European integration, including the commitments resulting from the status of an associated country stipulated in the European Digital Agenda 2020.

\section{References}

[1] BERTOT, J., JAEGER, P. and GRIMES, J.: Using ICTs to create a culture of transparency: E-government and social media as openness and anti-corruption tools for societies, in: Government Information Quarterly, 27, 2010, pp. 264-271.

[2] BURLACA, O. : Sistem de management al conținutului pentru Web, teză de doctorat, 2006, available at http://www.cnaa.acad.md/thesis/4735/ 
[3] COJOCARU, I., CUCIUREANU, G. and MORARU, O. : Instrumentul Bibliometric Naţional - sistem informati c performant, deschis, flexibil, scalabil, in Intellectus nr. 2, 2010, pp. 4455.

[4] EUROCRIS. Main features of CERIF, available at http://www.eurocris.org/cerif/mainfeatures-cerif

[5] EUROPEAN COMMISSION. Concept paper of the Digital Science vision, and its integration in the Horizon 2020 programme, 2013, available at https:/ec.europa.eu/digital-singlemarket/en/news/digital-science-horizon-2020

[6] LYON, L.: Transparency: the emerging third dimension of Open Science and Open Data, in: LIBER Quarterly. 25(4), pp.153-171, 2016, DOI: http://doi.org/10.18352/lq.10113

[7] TRANSPARENCY INTERNATIONAL. Corruption Perceptions Index, 2015, available at https://www.transparency.org/cpi2015/ 